\title{
A NOTE ON THE JACOBI CONDITION FOR PARAMETRIC PROBLEMS IN THE CALCULUS OF VARIATIONS*
}

BY M. R. HESTENES

1. Introduction. An elegant treatment of the Jacobi condition for parametric problems in $\left(y_{1} \cdots y_{n}\right)$-space has been given by Bliss. $\dagger$ He defines conjugate points in terms of solutions $\eta_{i}$ of the Jacobi equations which satisfy the relations $y_{i}^{\prime} \eta_{i}=0$. With the help of these solutions he establishes criteria for conjugate points in terms of the general solutions of the Jacobi equations. Since there are but $2 n-2$ linearly independent solutions $\eta_{i}$ satisfying the conditions $y_{i}^{\prime} \eta_{i}=0$, the treatment given by Bliss is quite different from that usually given for non-parametric problems. It is well known that the methods of Bliss are still applicable if the equation $y_{i}^{\prime} \eta_{i}=0$ is replaced by an equation such as $y_{i}{ }^{\prime} \eta_{i}{ }^{\prime}=0, y_{i}{ }^{\prime} \eta_{i}{ }^{\prime}=$ constant, and others. $\ddagger$ In the present paper we use the equation $y_{i}^{\prime} \eta_{i}^{\prime}=$ constant in defining conjugate points and obtain the same results as Bliss. The method used is, however, quite different from that of Bliss and has the advantage that it is almost identical with that usually given for the nonparametric problems. This follows because there are $2 n$ linearly independent solutions of the type considered in this paper. Other equations for which the method here used is still applicable are also discussed.

2. The Necessary Condition of Jacobi. The problem to be considered is that of minimizing an integral

$$
I=\int_{t_{1}}^{t_{2}} f\left(y_{1}, \cdots, y_{n}, y_{1}^{\prime}, \cdots, y_{n}^{\prime}\right) d t=\int_{t_{1}}^{t_{2}} f\left(y, y^{\prime}\right) d t
$$

\footnotetext{
* Presented to the Society, June 23, 1933.

$\dagger$ Bliss, Jacobi's condition for problems of the calculus of variations in parametric form, Transactions of this Society, vol. 17 (1916), pp. 195-206.

$\ddagger$ Graves, Discontinuous solutions in space problems of the calculus of variations, American Journal of Mathematics, vol. 52 (1930), p. 17. See also Wren, $A$ new theory of parametric problems in the calculus of variations, Contributions to the Calculus of Variations, 1930, The University of Chicago Press, pp. 175-
} 185. 
in a class of arcs $y_{i}=y_{i}(t),\left(t_{1} \leqq t \leqq t_{2} ; i=1, \cdots, n\right)$, which join two fixed points 1 and 2 in $\left(y_{1} \cdots y_{n}\right)$-space. The hypotheses upon which our analysis is based are those of Bliss.* The notations are also those of Bliss except that we use the tensor analysis summation convention instead of matrix notation.

A non-singular arc is one whose determinant

$$
\left|\begin{array}{cc}
f_{y_{i}^{\prime} y_{k^{\prime}}} & y_{i}^{\prime} \\
y_{k}^{\prime} & 0
\end{array}\right|
$$

is different from zero at each element $\left(y, y^{\prime}\right)$ on it A non-singular minimizing arc $E_{12}$ without corners is always an extremal arc. Moreover, the parameter $t$ can be chosen so that the functions $y_{i}(t)$ defining $E_{12}$ have continuous third derivatives. $\dagger$

We define a function $2 \omega\left(t, \eta, \eta^{\prime}\right)$ to be the function $\Omega\left(\eta, \eta^{\prime}\right)$ used by Bliss. Along a non-singular minimizing arc $E_{12}$ without corners the second variation of the integral $I$ is expressible in the form

$$
I_{2}=\int_{t_{1}}^{t_{2}} 2 \omega\left(t, \eta, \eta^{\prime}\right) d t,
$$

and this expression must be $\geqq 0$ for every set of admissible variations $\eta_{i}(t)$ which vanish at $t_{1}$ and $t_{2}$.

The Jacobi equations are the equations

$$
J_{i}(\eta)=(d / d t) \omega_{\eta_{i^{\prime}}}-\omega_{\eta_{i}}=0 .
$$

They satisfy the relations $y_{i}^{\prime} J_{i}(\eta) \equiv 0$ and hence are not independent. They are satisfied identically by the functions $\eta_{i}=\rho(t) y_{i}^{\prime}$, where $\rho(t)$ is an arbitrary function possessing continuous first derivatives. $\ddagger$

A special solution $\eta_{i}$ of the Jacobi equations is defined to be one which satisfies identically the equations $y_{i}^{\prime} \eta_{i}^{\prime}=$ constant. Every special solution satisfies with $\lambda \equiv 0$ the equations

$$
J_{i}(\eta)+\lambda y_{i}^{\prime}=0, \quad y_{i}^{\prime} \eta_{i}^{\prime \prime}+y_{i}^{\prime \prime} \eta_{i}^{\prime}=0 .
$$

Conversely every solution $\eta_{i}, \lambda$ of these equations has $y_{i}^{\prime} \eta_{i}^{\prime}$ $=$ constant, and $\lambda \equiv 0$ on account of the relations $y_{i}^{\prime} J_{i}(\eta) \equiv 0$,

* Loc. cit., p. 196.

$\dagger$ Bliss, loc. cit., pp. 197-198.

$\ddagger$ Bliss, loc. cit., p. 201. 
and hence the functions $\eta_{i}$ define special solutions of the Jacobi equations. Since $E_{12}$ is non-singular, equations (1) can be solved for the variables $\eta^{\prime \prime}, \lambda$. In the solutions the values for $\lambda$ and $\eta_{i}{ }^{\prime \prime}$ have the form $\lambda \equiv 0$, since $y_{i}{ }^{\prime} J_{i}(\eta) \equiv 0$, and

$$
\eta_{i}^{\prime \prime}=A_{i k}(t) \eta_{k}+B_{i k}(t) \eta_{k}^{\prime} \text {. }
$$

A solution $\eta_{i}$ of the Jacobi equations is therefore a special solution if and only if it also satisfies equations (2). The usual existence theorems for differential equations now tell us that through each element $\left(t_{0}, \eta_{i 0}, \eta_{i 0}{ }^{\prime}\right)$ there passes one and only one special solution $\eta_{i}$. Hence there exist $2 n$ linearly independent special solutions.

A point 3 is said to be conjugate to the point 1 on $E_{12}$ if there exists a special solution $\eta_{i}=u_{i}$ of the Jacobi equations whose functions $u_{i}(t)$ vanish at $t_{1}$ and $t_{3}$ but are not identically zero on $t_{1} t_{3}$. With this definition in mind the following necessary condition can be proved readily by the methods of Bliss.*

Theorem 1. The Necessary Condition of Jacobi. On a non-singular minimizing arc $E_{12}$ without corners there can be no point 3 conjugate to 1 between 1 and 2 .

In the proof of this theorem it is found by the methods of Bliss that if there were a special solution $u_{i}$ defining a point 3 conjugate to 1 between 1 and 2 on $E_{12}$, then this solution would take the values $u_{i}=0, u_{i}^{\prime}=c y_{i}^{\prime}$ at $t=t_{3}$. There is but one special solution taking these values at $t=t_{3}$, namely, the solution $u_{i}=\phi^{\prime}(a \phi+b) y_{i}^{\prime}$, where $a \phi^{\prime 2}\left(t_{3}\right)=c, b=-a \phi\left(t_{3}\right)$, and

$$
\phi(t)=\int_{t_{1}}^{t} d t /\left(y_{i}^{\prime} y_{i}^{\prime}\right)^{1 / 2},
$$

as is readily verified by substitution. This solution, however, vanishes at $t=t_{1}$ only in case $a=b=c=0$, that is, only in case $u_{i} \equiv 0$. It follows that there can be no point 3 conjugate to 1 between 1 and 2 on $E_{12}$, as was to be proved.

3. The Determination of Conjugate Points. Let $E_{12}$ be a nonsingular extremal arc whose parameter $t$ has been chosen so that the functions $y_{i}(t)$ defining $E_{12}$ have continuous third derivatives.

* Loc. cit., p. 200. 
Lemma 1. $A$ set of $2 n$ special solutions $u_{i s},(s=1, \cdots, 2 n)$, of the Jacobi equations have a determinant

$$
\left|\begin{array}{l}
u_{i s}(t) \\
u_{i s}^{\prime}(t)
\end{array}\right|
$$

which is either identically zero or else everywhere different from zero.

This result is a consequence of well known theorems on linear homogeneous differential equations of the second order applied to equations (2). A further consequence of these theorems is that every special solution $u_{i}$ is expressible linearly with constant coefficients in terms of $2 n$ special solutions $u_{i s}$ whose determinant (4) is different from zero. The following two lemmas can now be established by the usual methods.*

Lemma 2. The points 3 conjugate to 1 on $E_{12}$ are determined by the zeros $t_{3} \neq t_{1}$ of the determinant

$$
\Delta\left(t, t_{1}\right)=\left|\begin{array}{c}
u_{i s}(t) \\
u_{i s}\left(t_{1}\right)
\end{array}\right|
$$

formed for $2 n$ special solutions $u_{i s}$ of the Jacobi equations whose determinant (4) is different from zero.

LEMMA 3. If the functions $u_{i k}(t),(k=1, \cdots, n)$, define $n$ linearly independent special solutions of the Jacobi equations which vanish at $t=t_{1}$, then the points 3 conjugate to 1 on $E_{12}$ are determined by the zeros $t_{3} \neq t_{1}$ of the determinant $\left|u_{i k}(t)\right|$.

We also need the further lemma:

LEMMA 4. Every solution $\eta_{i}$ of the Jacobi equations has associated with it a two-parameter family of special solutions

$$
u_{i}=\eta_{i}-\rho y_{i}^{\prime} \text {, }
$$

where

$$
\rho=\phi^{\prime}\left(a \phi+b+\int_{t_{1}}^{t} y_{i}^{\prime} \eta_{i}^{\prime} \phi^{\prime} d t\right),
$$

$\phi(t)$ is the function (3), and $a, b$ are arbitrary constants.

* See, for example, Bliss, The problem of Lagrange in the calculus of variations, American Journal of Mathematics, vol. 52 (1930), pp. 727-728. 
This result is readily obtained by solving for $\rho$ in the equations

$$
y_{i}^{\prime} u_{i}^{\prime}=y_{i}^{\prime} \eta_{i}^{\prime}-\rho^{\prime} y_{i}^{\prime} y_{i}^{\prime}-\rho y_{i}^{\prime \prime} y_{i}^{\prime}=-a .
$$

From Lemma 4 , with $a=b=0$, it follows that every set of $2 n-2$ solutions $u_{i q},(q=1, \cdots, 2 n-2)$, of the Jacobi equations has associated with it a set of $2 n-2$ two-parameter families of special solutions $v_{i q}=u_{i q}-\rho_{q} y_{i}^{\prime}$. Moreover, the functions $\phi \phi^{\prime} y_{i}^{\prime}$, $\phi^{\prime} y_{i}{ }^{\prime}$, where $\phi$ is the function (3), also define special solutions, as is easily seen by substitution. The determinants (4) and (5) formed for the $2 n$ special solutions $v_{i q}, \phi \phi^{\prime} y_{i}{ }^{\prime}, \phi^{\prime} y_{i}{ }^{\prime}$ are equal, respectively, to

where

$$
\phi^{\prime 3} d(t), \quad \phi(t) \phi^{\prime}(t) \phi^{\prime}\left(t_{1}\right) D\left(t, t_{1}\right),
$$

$$
\begin{aligned}
d(t) & =\left|\begin{array}{lcc}
u_{i q}(t) & 0 & y_{i}^{\prime}(t) \\
u_{i q}^{\prime}(t) & y_{i}^{\prime}(t) & y_{i}^{\prime \prime}(t)
\end{array}\right|, \\
D\left(t, t_{1}\right) & =\left|\begin{array}{lcc}
u_{i q}(t) & y_{i}^{\prime}(t) & 0 \\
u_{i q}\left(t_{1}\right) & 0 & y_{i}^{\prime}\left(t_{1}\right)
\end{array}\right| .
\end{aligned}
$$

Since $\phi^{\prime}>0$, the zeros of $D\left(t, t_{1}\right)$ are identical with those of $\Delta\left(t, t_{1}\right)$. Hence, by the use of Lemmas 1 and 2 , we obtain the following theorem of Bliss.

THEOREM 2. The determinant $d(t)$ formed for $2 n-2$ solutions $u_{i q},(q=1, \cdots, 2 n-2)$, of the Jacobi equations is either identically zero or else everywhere different from zero. Moreover, the points 3 conjugate to 1 on $E_{12}$ are determined by the zeros $t_{3} \neq t_{1}$ of the determinant $D\left(t, t_{1}\right)$ formed for $2 n-2$ solutions $u_{i q}$ whose determinant $d(t)$ is different from zero.

We can also prove the further criterion for conjugate points given by Bliss.

THEOREM 3. If the functions $u_{i r}(t),(r=1, \cdots, n-1)$, define $n-1$ solutions of the Jacobi equations whose matrix

$$
\left\|\begin{array}{ccc}
u_{i r} & 0 & y_{i}^{\prime} \\
u_{i r}^{\prime} & y_{i}^{\prime} & y_{i}^{\prime \prime}
\end{array}\right\|
$$

has rank $n+1$ at $t=t_{1}$, while the determinant

$$
D(t)=\left|u_{i r}(t) \quad y_{i}^{\prime}(t)\right|
$$


has rank 1 at $t=t_{1}$, then the points 3 conjugate to 1 on $E_{12}$ are determined by the zeros $t_{3} \neq t_{1}$ of $D(t)$.

In order to prove this we note that since $D\left(t_{1}\right)$ has rank 1 , there exist constants $c_{r}$ such that $u_{i r}-c_{r} y_{i}^{\prime}=0$ at $t=t_{1}$. By taking $a=0$ and $b=c_{r} / \phi^{\prime}\left(t_{1}\right)$ in Lemma 4 , it is found that the $n-1$ solutions $u_{i r}$ have associated with them $n-1$ special solutions $v_{i r}=u_{i r}-\rho_{r} y_{i}^{\prime}$ such that $\rho_{r}\left(t_{1}\right)=c_{r}$ and hence such that $v_{i r}\left(t_{1}\right)=0$. As above, it is easily seen by substitution that the functions $v_{i}=\phi^{\prime} \phi y_{i}^{\prime}$ define a special solution having $v_{i}\left(t_{1}\right)=0$. The $n$ special solutions $v_{i r}, v_{i}$ are linearly independent since the matrix (7) has rank $n+1$. Moreover, the determinant $\left|v_{i r} v_{i}\right|$ is equal to $\phi \phi^{\prime} D(t)$. Its zeros, therefore, coincide with those of $D(t)$ since $\phi^{\prime}(t)>0$. The theorem now follows from Lemma 3 .

4. Other Types of Special Solutions. In $\$ \$ 2$ and 3 above, the equation $y_{i}^{\prime} \eta_{i}^{\prime}=$ constant was used in order to define special solutions. We could use instead any equation which is equivalent to one of the form

$$
y_{i}^{\prime} \eta_{i}^{\prime \prime}+C_{i}(t) \eta_{i}^{\prime}+D_{i}(t) \eta_{i}=0
$$

and which has a two-parameter family of solutions of the form $\eta_{i}=\left[a \rho_{1}(t)+b \rho_{2}(t)\right] y_{i}^{\prime}(t)$ with the property that for every pair of constants $(a, b) \neq(0,0)$ the expression $a \rho_{1}+b \rho_{2}$ vanishes at most once on the interval under consideration. The equations $y_{i}^{\prime} \eta_{i}=a t+b$ and $y_{i}^{\prime} \eta_{i}^{\prime}=a y_{i}^{\prime} y_{i}^{\prime}$, for example, have this property, as one readily verifies.

It is interesting to note that the special solutions used in $\$ \S$ 2 and 3 above are closely related to extremal families having arc length as their parameter. If the functions

$$
y_{i}=y_{i}\left(t, c_{1}, \cdots, c_{2 n-2}\right)
$$

define a $(2 n-2)$-parameter family of extremals containing $E_{12}$ for $c_{q}=c_{q 0},(q=1, \cdots, 2 n-2)$, and having arc length as parameter, then along $E_{12}$ the derivatives $y_{i a}, y_{i b}, y_{i c_{q}}$ of the functions $y_{i}\left(a t+b, c_{q}\right)$ form $2 n$ special solutions having the properties described in Lemmas 1 and 2 . In order to prove that these derivatives define special solutions one needs only to differentiate the identity $y_{i}^{\prime} y_{i}^{\prime}=a^{2}$ with respect to $a, b, c_{q}$ and set $c_{q}=c_{q 0}$.

The University of Chicago 